UDC 616.831.31-009.24

DOI: $10.15587 / 2519-4798.2020 .217557$

\title{
ANALYSIS OF THE ROLE OF VIRAL ENCEPHALITIS IN THE DEVELOPMENT OF EPILEPTIC SEIZURES AND EPILEPSY
}

\author{
T. Litovchenko, O. Bilchenko, V. Malakhov, O. Dubenko, O. Sukhonosova, V. Bokatueva, \\ M. Markova, V. Nikonov
}

\begin{abstract}
The aim - to determine the role of viral lesions of the central nervous system (CNS) in the development of epileptic seizures and epilepsy.

Materials and methods: analysis of scientific literature data. The review discusses the role of non-epidemic viral encephalitis and human immunodeficiency virus in the development of acute epileptic seizures and epilepsy; epidemiological data and risk factors.

Results. Viral infections are often complicated by acute (early) epileptic seizures and causes the risk of further epilepsy. The mechanisms of development of early and late seizures differ. Except herpetic encephalitis, in which risk of epilepsy in early seizures is up to $60 \%$, the risk of late seizures in other viral encephalitis hasn't been determined. Timely treatment of viral infections and early seizures reduces the risk of epilepsy. Treatment of epilepsy with infectious lesions of the central nervous system and other types of symptomatic epilepsy is similar, and the antiepileptic drug is determined by the semiology of seizures. The interaction between it and antiinfective drugs can change the concentration in the blood of each of them, which will lead to reduced effectiveness or toxicity. This is especially important for individuals with human immunodeficiency virus infection in whom antiretroviral drugs have significant interactions with antiepileptic drugs. Since epileptic activity of the brain is associated with the severity and complications of the underlying infection, early aggressive therapy prevents the development of late seizures and epilepsy.

Conclusions. CNS infections account for $15 \%$ of all newly diagnosed symptomatic epileptic seizures. The 20-year risk of unprovoked seizures and epilepsy after CNS infections ranges from $2.4 \%$ to $22 \%$. The risk depends on the etiology, location of the lesion and the severity; it is high in herpes-associated encephalitis
\end{abstract}

Keywords: central nervous system, seizures, epilepsy, encephalitis, viral infections, pathogenesis, risk factors, forecasting, treatment, review

Copyright (C 2020, T. Litovchenko, O. Bilchenko, V. Malakhov, O. Dubenko, O. Sukhonosova, V. Bokatueva, M. Markova, V. Nikonov. This is an open access article under the CC BY license (http://creativecommons.org/licenses/by/4.0).

\section{Introduction}

According to WHO experts, there are more than 50 million people with epilepsy in the world, with 40 million living in low-income and developing countries [1], and about half of them are children.

Central nervous system (CNS) infections account for $15 \%$ of all cases of new-onset symptomatic seizures [2]. And the 20-year risk of unprovoked seizures and epilepsy after CNS infections is from $2.4 \%$ to $22 \%$. The risk depends on the etiology of the infection, the location of the lesion and severity. There is a high risk of developing epilepsy in herpetic encephalitis. The risk increases significantly with the onset of seizures in the acute period of CNS infections and, especially, with the development of status epilepticus [2].

According to a number of studies, the overall risk of developing epilepsy due to CNS infections is $3 \%$, but the incidence varies significantly in different regions due to the prevalence of different patterns of CNS infections in the world $[2,3]$. The incidence, course and consequences of epilepsy due to acute CNS infections have not been systematized in studies to date.

Central nervous system (CNS) infections are the leading cause of seizures and acquired epilepsy in a number of regions of the world $[4,5]$. Acute seizures are common in severe meningitis, viral encephalitis, malaria, and neurocysticercosis, and are associated with increased mortality and morbidity, including the development of epilepsy. Neuronal excitability, secondary to proinflammatory signals caused by CNS infections, is an important general mechanism of seizure generation, in addition to other specific mechanisms [6].

Seizures associated with CNS infections can be both acute symptomatic and unprovoked epileptic seizures. Acute symptomatic attacks occur in close time with an acute CNS infection of $31 \%$, usually within the first 7 days, sometimes even later, but always during the infectious process and usually do not recur; however, there is an increased risk of developing further epilepsy [7]. The risk of developing epilepsy after CNS infections ranges from $6.8 \%$ to $8.3 \%$ (in developed countries) and is much higher in countries with low living standards (so in Central Africa it reaches $26 \%$ ).

Large epidemiological studies show that infections account for 2-3\% of the etiological causes of newly diagnosed epilepsy [3], and almost half of them are viral infections. Viral infections of the CNS can occur in the form of mild, often subclinical meningitis, as well as severe acute viral encephalitis, mortality in which reaches $70 \%$ in the absence of adequate treatment. The risk of developing epilepsy as a result of viral encephalitis increases $7-10$ times. This risk is 22 -fold higher than the 
premorbid risk in patients who have had early attacks in the acute period of infection. In most cases, seizures develop within 5 years after an acute infection, but there may be cases of epilepsy after 15 years. There are no reliable data on the increased risk of epilepsy after viral meningitis $[8,9]$.

Patients with early seizures in encephalitis have a cumulative risk of developing epilepsy of $10 \%$ up to 5 years and $22 \%$ up to 20 years; this risk is approximately twice as high as in patients with encephalitis who did not have early seizures [10-12]. Patients with postencephalitic epilepsy (PE) often develop resistant multifocal seizures [13-15].

\section{Sporadic (nonspecific) changes in the context of the development of epileptic seizures and epilepsy \\ 2.1. Viral encephalitis}

Viral encephalitis is caused by many known and unidentified viral agents. By this term we mean acute viral encephalitis, post-infectious encephalomyelitis, slow viral CNS infections and chronic degenerative CNS diseases, where a viral etiology is assumed [16].

Viral encephalitis is a common cause of epileptic seizures, especially in children. More than 100 viruses can cause encephalitis; however, due to the nonspecific nature of the disease and the lack of reliable diagnostic tools, the etiology in most cases remains undetected $[17,18]$.

The prevalence of viral encephalitis varies considerably depending on the geographical region. In Western European developed countries, there are about 10.5 cases per 100,000 in children and 2.2 cases per 100,000 adults [19]. But rates are much higher in tropical regions and in countries with low living standards. Viral encephalitis can occur in epidemics or be sporadic. Most epidemics are associated with arboviruses and have a distinct geographical distribution both in and within countries. Sporadic (nonspecific) encephalitis is of considerable interest in Ukraine.

Sporadic forms of viral encephalitis: herpesvirus (herpes simplex 1 and 2 types, herpes varicella-zoster, human herpesvirus 6, Epstein-Barr virus, cytomegalovirus), RNA viruses (measles, mumps, influenza), enteroviruses (coxsackie viruses, echoviruses). Herpesvirus encephalitis, which is the most common sporadic encephalitis, has a high mortality and severe consequences.

\subsection{Clinical signs of the disease}

Viral encephalitis is characterized by an acute onset, significant hyperthermia, headache, nausea, drowsiness and other non-specific symptoms characteristic of an infectious disease, as well as symptoms of leptomeningeal irritation. The development of viral infection is accompanied by edema of the brain parenchyma, neuronal and glial degeneration, inflammatory infiltration, necrotic changes. Early neurological symptoms may be manifested by behavioural disorders (agitation, embarrassment, irritability, speech disorders, dysphasia), hemiparesis develops in a third of cases. Often only when a patient develops epileptic seizures, stupor or coma is diagnosed with a serious illness. Focal seizures with / without impaired awareness of temporal localization are most characteristic of herpes simplex type $1[2,3]$.
The specificity of the viral lesion is detected in the cerebrospinal fluid by PCR (polymerase chain reaction). Herpesvirus lesions (HSV-1 and HSV-2), cytomegalovirus (CMV), chickenpox virus (VZV), Epstein-Barr virus (EBV), herpesvirus type 6 (HHV-6) are likely to be diagnosed by PCR.

If early differential PCR diagnosis of viral and bacterial lesions is not possible and a characteristic clinical picture is present, early treatment with a combination of antiviral drugs (acyclovir) and broad-spectrum antibiotics is recommended.

\subsection{Herpesvirus infections}

Herpes simplex virus (HSV-1 and HSV-2) is the most common cause of sporadic (non-epidemic) encephalitis. HSV-1 encephalitis is the most common form in children older than 3 months and in adults, accounting for more than $20 \%$ of all viral encephalitis. The lesion predominates in the temporal and fronto-temporal areas. HSV-2 prevails in infants and is transmitted from an infected mother during childbirth, genital HSV-2 is detected in $85 \%$ of such cases. Skin lesions are a sign of such infection in newborns in the first week of life, but isolated herpesvirus encephalitis is possible in 2 weeks after birth, characterized by generalized lesions of the brain [20]. In adults, HSV-2 encephalitis is more likely to develop in the presence of immunosuppression.

In most cases, HSV-1 encephalitis is the result of reactivation of a latent infection, especially in the elderly. Primary cases of infection are found in $1 / 3$ of young patients (up to 20 years). The reasons for reactivation of latent infection in immunocompetent patients are unknown.

Herpes simplex virus type 1 is the most common cause of sporadic encephalitis and is the cause of seizures in more than $50 \%$ of patients. The susceptibility of herpes simplex virus type 1 to lesions of the mesial temporal lobe, including the hippocampus, explains the high frequency of attacks. Seizures are often focal, and recurrent lateralized epileptiform discharges (PLEDs) on the EEG are common in adults. Children may have atypical traits, generalized seizures and lack of PLEDs [2, 21]. Behavioural disorders are common in older children and adults. Herpes simplex virus type 1 also leads to latent and persistent infection with late recurrence and reactivation. Late unprovoked seizures and epilepsy occur in 42-60\% of patients and are often resistant.

Neuroimaging (MRI) data often do not detect pathology in the first week of the disease. Characteristic MRI signs are increased signal intensity, edema, hemorrhage, lesions of grey/white matter in the temporal lobe, islet cortex, cingulate cortex. Diffuse-weighted MRI can be used to detect early lesions [22].

Electroencephalographic changes (EEG) are usually presented in most patients with encephalitis, but only $40 \%$ of patients have acute seizures. The most common is diffuse high-amplitude deceleration in the temporal regions, the possible presence of focal temporal or lateralized delta activity with PLEDS, repeated at intervals of 1-5 seconds, mainly in the temporal regions for 4 weeks from the onset of the disease. Such complexes can be unilateral or bilateral, synchronous / asynchronous, and usually accompany the spread of pathology on 
the affected side. Such changes may be one of the diagnostic signs of HSV encephalitis.

Treatment of epileptic seizures in the acute period largely determines the further prognosis, as uncontrolled seizures in the acute period are factors of unfavourable prognosis [23, 24].

Untreated HSV encephalitis leads to death in $70 \%$ of cases, with proper treatment an average of $20 \%$ of cases, and early treatment reduces mortality to $10 \%$. Epilepsy develops more often in the presence of lesions of the temporal lobe. The attacks are mostly focal with/without evolution into bilateral tonic-clonic seizures. In a significant number of cases, epileptic seizures are resistant to antiepileptic drugs (AED), in the presence of an operable focus, surgical treatment is successful. There are studies that indicate the possibility of prolonged persistence of herpes virus infection (chronic herpes virus viral encephalitis) in patients with resistant epilepsy.

Epstein-Barr virus (EBV) is very common in the human population, with studies showing that most people are infected before the age of 40 . In the pediatric population, this virus rarely causes clinical manifestations, adolescents in 1/3-1/2 cases develop infectious mononucleosis.

Involvement of the CNS in the infectious process is quite rare, but can lead to encephalitis, aseptic meningitis, meningoencephalitis. EBV is the cause of $5 \%$ of all cases of viral encephalitis, more common in children or in immunosuppressed adults. Usually patients with EBV encephalitis do not have symptoms typical of infectious mononucleosis. The disease is manifested by nonspecific prodrome and convulsions [16, 25]. Epileptic seizures are noted in $36-48 \%$ of cases in the pediatric population [26], the development of status epilepticus in the acute stage of the disease is uncharacteristic. Epilepsy, according to many researchers, develops in $1 / 3$ of cases.

Human herpes virus type 6 (HHV-6), as well as EBV virus, is widespread, the primary infection occurs in childhood with classic manifestations in the form of fever, weakness, cough, classic rash (roseola infantum), the symptoms are usually transient. However, HHV-6 is often associated with febrile seizures in children under 5 years of age, which occur in $5 \%$ of infected children, HHV-6 infection is found in $1 / 3$ of children under 2 years of age with febrile seizures. In the future, such children are diagnosed with epilepsy in only $2 \%$ of cases. HHV-6 encephalitis mostly develops in the presence of immunosuppression (patients after transplantation, etc.) and has a severe course and unfavourable prognosis (mortality up to $50 \%$ ) [2, 16].

Cytomegalovirus (CMV) encephalitis is one of the most common opportunistic infections in HIVinfected patients and is rarely diagnosed in immunocompetent individuals. The disease is manifested by fever, headache, confusion, dysphasia, cranial nerve palsy, epileptic seizures, possible coma. In the acute stage, MRI often reveals periventricular edema and signal elevation in the frontal and parietal regions on T2-weighted images. In immunocompetent individuals, the persistence of epileptic seizures after recovery is atypical. Patients with immune system damage often develop epilepsy, and children with symptomatic congenital CMV in 20-25\% of cases [2, 16].

Chickenpox virus (varicella zoster virus - VZV) is the second most common cause of viral encephalitis, diagnosed in 1:2000 infected individuals with the same frequency in adults and children, with a high risk of mortality - up to $15 \%$ even in immunocompetent patients. Encephalitis can develop as a component of clinical manifestations of chickenpox with classic encephalitic manifestations in a week after the appearance of vesicular rash, as well as reactivation of latent infection (years after chickenpox) more often in immunosuppressed patients. Epileptic seizures are the most common clinical manifestation and occur in 29-52\% in the acute stage of the disease. Two main mechanisms of damage in VZVencephalitis are determined - it is a direct damaging effect of the virus and the development of autoimmune encephalitis in response to viral aggression [27]. MRI examination reveals multiple ischemic foci (vasculitis) with increased signal subcortically [22].

\subsection{RNA viruses}

Measles is often involved in the pathological process of the CNS, the measles virus can cause acute encephalitis, subacute sclerosing panencephalitis more often in school-age children. Encephalitis develops in 1 in 1000 cases of measles usually a week after the onset of the disease. The risk of mortality is up to $15 \%$, and severe neurological consequences - epilepsy, cognitive deficits are observed in 1/3 of cases [28, 29]. In CSR at encephalitis moderate pleocytosis is defined, antibodies to a measles virus are absent.

Subacute sclerosing panencephalitis is a rare progressive degenerative disease that develops in 1 in 100,000 cases of measles. It develops in children and young adults on average 7 years after measles. The disease progresses, the course is very variable, but death in most cases occurs in 1-3 years. The clinical picture is characterized by severe myoclonic seizures, progressive dementia and motor deficiency. In the CSF (cerebrospinal fluid) it is determined by a high level of antibodies to the measles virus. Significant changes in white matter and severe cerebral atrophy are indicative of MRI $[18,22]$.

Infectious mumps. Meningitis due to mumps accounts for $10 \%$ of all infected, and the severity of the primary disease (mumps) has almost no effect on the development of CNS damage. The disease is characterized by severe course, high mortality and the development of neurological consequences. Mumps is a rare complication $(0.1 \%)$. The first symptoms are usually epileptic seizures, disturbances of consciousness and focal neurological symptoms, which are more common in adults [30].

Influenza. Both strains (A and B) may be accompanied by febrile seizures and the risk of developing encephalitis, but strain A has a higher risk of neurological consequences. Virus A is associated with the development of febrile seizures in children in $20 \%$. Encephalitis is an atypical complication of virus A. The risk of morbidity and mortality during epidemics is significant and is $27-50 \%$ and $31 \%$, respectively. Vaccination reduces the risk of developing febrile seizures. 
Enteroviruses. The group of human enteroviruses includes polioviruses, coxsackie viruses, enterovirus 71, transmitted by fecal-oral and hematogenous routes. Usually viral replication occurs in the gastrointestinal tract, but during the viremic phase may damage other systems (CNS, myocardium, meninges, skin), the incubation period is from 2 to 40 days. All human enteroviruses are associated with aseptic meningitis. Frequent complications of viral infection in children are febrile seizures. Treatment of enterovirus infection is carried out with a specific immunoglobulin, but it has low permeability to the CNS. Severe neurological consequences and epilepsy rarely develop $[2,16]$.

\subsection{Mechanisms of development of epileptic seizures and epilepsy}

The mechanisms of development of epileptic seizures and epilepsy after CNS infections are not well established. Structural lesions such as cortical necrosis with herpes simplex virus, myocardial infarction, hypoxicischemic trauma in cerebral malaria and gliosis can be epileptogenic foci. It is also believed that prolonged stimulation of pro-inflammatory signals by both chronic inflammation and seizures themselves can lead to a residual pathological condition, such as damaged bloodbrain barrier, neuronal death and persistent hyperexcitability of neurons - all this may contribute to epileptogenesis [9].

The inflammatory cascade destroys the bloodbrain barrier, cerebral edema, increased intracranial pressure, cerebral hemorrhage and heart attacks, which contributes to the development of epileptic seizures. Hyperthermia associated with various CNS infections can in itself lead to hyperexcitability of neurons. Other factors including fluid and electrolyte imbalance, hypoglycemia, vasculitis, thrombosis, and infarction are the main mechanisms in individuals with tuberculous meningitis and fungal CNS infections. The formation of an abscess in purulent, tuberculous and fungal infections increases the susceptibility to seizures. Neurotoxins such as quinolinic acid, the production of autoantibodies to glutamate and $\mathrm{N}$-methyl-D-aspartate receptors, changes in calcium and potassium channel function, and the effects of antiphospholipid, anticardiolipin, and antinuclear antibodies are considered to be other mechanisms responsible for seizures [31].

Thus, viral infections are often complicated by epileptic seizures in the acute phase of the disease (early seizures) and lead to an increased risk of developing epilepsy in the future. The mechanisms of development of early and late seizures are different. With the exception of herpes simplex encephalitis, in which the risk of epilepsy in the presence of early seizures is up to $60 \%$, the risk of late seizures in other viral encephalitis is not well established. Timely treatment of viral infection and early seizures can reduce the risk of epilepsy $[9,22]$.

The risk of postencephalitic epilepsy increases at 22 times in individuals with seizures in the acute stage of encephalitis compared with 10 times in individuals without acute seizures $[32,10]$. Most unprovoked seizures occur within the first 5 years after an episode of encephalitis, but the risk of seizures persists for 20 years or more.
Patients with status epilepticus, slow background activity, and multifocal EEG adhesions, individuals with HSV1 encephalitis, have a significantly higher risk of developing resistant epilepsy [15, 33-35].

\section{Principles of treatment of epileptic seizures and epilepsy on the background of a viral infection \\ Treatment of epilepsy due to an infectious le- sion of the CNS is similar to other symptomatic epi- lepsies, and the antiepileptic drug is selected accord- ing to the semiology of seizures. But before starting treatment, a number of specific questions need to be addressed:}

(1) What is the interaction between AED and antiinfective therapy?

(2) Can early treatment of an underlying CNS infection prevent or alter the course of seizures and epilepsy?

(3) Are there other treatments to reduce the frequency of seizures and epilepsy in CNS infections?

(4) What is the optimal duration of AED therapy?

Interactions between AEDs and anti-infection therapy can significantly alter the level of each drug in the blood, leading to either reduced efficacy or toxicity. Since seizures and epilepsy are often associated with the severity and complications of the underlying infection, it seems logical that early aggressive therapy can prevent the development of late seizures and epilepsy. However, there are no systematic studies to confirm this. Since seizure generation in most CNS infections is associated with an inflammatory response, it is possible to reduce seizures with anti-inflammatory drugs [36, 37].

The duration of antiepileptic therapy has not been discussed once, but there are no evidence-based recommendations. In acute bacterial meningitis, early attacks do not require long-term AEDs; however, late attacks are often associated with a major complication and are prone to recurrence. Thus, the duration of AED therapy depends on several factors and requires individualization $[9,37-39]$.

\section{Conclusions}

1. Viral infections are often complicated by epileptic seizures in the acute phase of the disease (early seizures) and lead to an increased risk of developing epilepsy in the future. CNS infections lead to $15 \%$ of all cases of newly diagnosed symptomatic seizures. The 20 -year risk of unprovoked seizures and epilepsy after CNS infections ranges from $2.4 \%$ to $22 \%$. The risk depends on the etiology of the infection, the location of the lesion and severity; it is high in herpetic encephalitis.

2. The mechanisms of development of early and late seizures differ. Excluding herpetic encephalitis, when the risk of epilepsy in early seizures is up to $60 \%$, the risk of developing late seizures in other viral encephalitis has not been definitively established.

3. Timely treatment of viral infection and early seizures can reduce the risk of developing epilepsy. Treatment of epilepsy due to an infectious lesion of the CNS is similar to other symptomatic epilepsies, and the antiepileptic drug is selected according to the semiology of seizures. Interactions between it and antiinfective drugs can significantly change the concentration in the blood of each of them, which will lead to 
reduced efficiency or toxicity. This is especially important for people with human immunodeficiency virus infection, in whom antiretroviral drugs have significant interactions with antiepileptic drugs. Because seizures and epilepsy are associated with the severity and complications of the underlying infection, early aggressive therapy prevents the development of late seizures and epilepsy.

\section{Conflicts of interest}

The authors declare that they have no conflicts of interest.

\section{References}

1. World Health Organization. Epilepsy fact sheet no. 999 January 2009. Available at: thttp://www.who.int/mediacentre/ factsheets/fs999/en/index.html Last accessed: 23.10.2020

2. Shorvon, S. D., Perukka, E., Engel, J. (Eds.) (2009). The treatment of epilepsy. Blackwell Publishing Ltd, 1075. doi: http://doi.org/10.1002/9781444316667

3. Sander, J. W., Hart, Y. M., Johnson, A. I., Shorvon, S. D. (1990). Medical science. National general practice Study of Epilepsy: newly diagnosed epileptic seizures in general population. Lancet, 336 (8726), 1267-1271. doi: http://doi.org/10.1016/0140-6736(90)92959-1

4. Bhalla, D., Godet, B., Druet-Cabanac, M., Preux, P.-M. (2011). Etiologies of epilepsy: a comprehensive review. Expert Review of Neurotherapeutics, 11 (6), 861-876. doi: http://doi.org/10.1586/ern.11.51

5. Fujiwara, T., Shigematsu, H. (2004). Etiologic factors and clinical features of symptomatic epilepsy: Focus on pediatric cases. Psychiatry and Clinical Neurosciences, 58 (3), S9-S12. doi: http://doi.org/10.1111/j.1440-1819.2004.01244_3.x

6. Singh, T. D., Fugate, J. E., Hocker, S. E., Rabinstein, A. A. (2014). Postencephalitic epilepsy: Clinical characteristics and predictors. Epilepsia, 56 (1), 133-138. doi: http://doi.org/10.1111/epi.12879

7. Ziai, W. C., Lewin, J. J. (2008). Update in the Diagnosis and Management of Central Nervous System Infections. Neurologic Clinics, 26 (2), 427-468. doi: http://doi.org/10.1016/j.ncl.2008.03.013

8. Duncan, J. S., Shorvon, S. D., Fish, D. R. (1995). Clinical Epilepsy. London: Churchill Livingstone, 540.

9. Shorvon, S. D., Andermann, F., Guerrini, R. (2011). The Causes of Epilepsy. Cambridge: Cambridge Univers. press, 787. doi: http://doi.org/10.1017/cbo9780511921001

10. Annegers, J. F., Hauser, W. A., Beghi, E., Nicolosi, A., Kurland, L. T. (1988). The risk of unprovoked seizures after encephalitis and meningitis. Neurology, 38 (9), 1407-1410. doi: http://doi.org/10.1212/wnl.38.9.1407

11. Cardarelli, W. J., Smith, B. J. (2010). The burden of epilepsy to patients and payers. The American Journal of Managed Care, 16, S331-S336.

12. Marks, D. A., Kim, J., Spencer, D. D., Spencer, S. S. (1992). Characteristics of intractable seizures following meningitis and encephalitis. Neurology, 42 (8), 1513-1518. doi: http://doi.org/10.1212/wnl.42.8.1513

13. Nieto Barrera, M. (2002). Aspectos clínicos, neurorradiológicos y evolutivos de las epilepsias catastróficas postencefalíticas. Revista de Neurología, 35 (S1), 30-38. doi: http://doi.org/10.33588/rn.35s1.2002183

14. Trinka, E., Dubeau, F., Andermann, F. Bastos, A., Hui, A., Li, L. M. et. al. (2000). Clinical findings, imaging characteristics and outcome in catastrophic post-encephalitic epilepsy. Epileptic Disord, 2, 153-162.

15. Chen, Y.-J., Fang, P.-C., Chow, J. C. (2006). Clinical Characteristics and Prognostic Factors of Postencephalitic Epilepsy in Children. Journal of Child Neurology, 21 (12), 1047-1051. doi: http://doi.org/10.1177/7010.2006.00223

16. Zelano, J., Westman, G. (2020). Epilepsy after brain infections in adults: A register-based population-wide study. Neurology. doi: http://doi.org/10.1212/wnl.0000000000010954

17. Granerod, J., Crowcroft, N. S. (2007). The epidemiology of acute encephalitis. Neuropsychological Rehabilitation, 17 (4-5), 406-428. doi: http://doi.org/10.1080/09602010600989620

18. Singhi, P. (2011). Infectious causes of seizures and epilepsy in the developing world. Developmental Medicine \& Child Neurology, 53 (7), 600-609. doi: http://doi.org/10.1111/j.1469-8749.2011.03928.x

19. Jmor, F., Emsley, H. C., Fischer, M., Solomon, T., Lewthwaite, P. (2008). The incidence of acute encephalitis syndrome in Western industrialised and tropical countries. Virology Journal, 5 (1), 134-136. doi: http://doi.org/10.1186/1743-422x-5-134

20. Whitley, R. J. (2006). Herpes simplex encephalitis: Adolescents and adults. Antiviral Research, 71 (2-3), 141-148. doi: http://doi.org/10.1016/j.antiviral.2006.04.002

21. Elbers, J. M., Bitnun, A., Richardson, S. E., Ford-Jones, E. L., Tellier, R., Wald, R. M. et. al. (2007). A 12-Year Prospective Study of Childhood Herpes Simplex Encephalitis: Is There a Broader Spectrum of Disease? Pediatrics, 119 (2), e399-e407. doi: http://doi.org/10.1542/peds.2006-1494

22. Solomon, T., Hart, I. J., Beeching, N. J. (2007). Viral encephalitis: a clinical guide. Practical Neurology, 7, $288-305$. doi: http://doi.org/10.1136/jnnp.2007.129098

23. Yamada, S., Kameyama, T., Nagaya, S., Hashizume, Y., Yoshida, M. (2003). Relapsing herpes simplex encephalitis: pathological confirmation of viral reactivation. Journal of Neurology, Neurosurgery \& Psychiatry, 74 (2), $262-264$. doi: http://doi.org/10.1136/jnnp.74.2.262

24. Kim, M. A., Park, K. M., Kim, S. E., Oh, M. K. (2008). Acute symptomatic seizures in CNS infection. European Journal of Neurology, 15, 38-41. doi: http://doi.org/10.1111/j.1468-1331.2007.01996.x

25. Doja, A., Bitnun, A., Ford Jones, E. L., Richardson, S., Tellier, R., Petric, M. et. al. (2006). Pediatric Epstein-Barr Virus - Associated Encephalitis: 10-Year Review. Journal of Child Neurology, 21 (5), 384-391. doi: http://doi.org/10.1177/08830738060210051101

26. Hung, K. L., Liao, H. T., Tsai, M. L. (2000). Epstein-Barr virus encephalitis in children. Acta Paediatr Taiwan, 41, 140-146.

27. Häusler, M., Schaade, L., Kemény, S., Schweizer, K., Schoenmackers, C., Ramaekers, V. T. (2002). Encephalitis related to primary varicella-zoster virus infection in immunocompetent children. Journal of the Neurological Sciences, 195 (2), $111-116$. doi: http://doi.org/10.1016/s0022-510x(02)00017-5

28. Perry, R. T., Halsey, N. A. (2004). The Clinical Significance of Measles: A Review. The Journal of Infectious Diseases, 189 (1), 4-16. doi: http://doi.org/10.1086/377712

29. Cherry, J., Demmler-Harrison, G., Kaplan, Sh., Steinbach, W., Hotez, P. (2017). Feigin and Cherry's Textbook of Pediatric Infectious Diseases. Elsevier, 3000.

30. Hviid, A., Rubin, S., Mühlemann, K. (2008). Mumps. The Lancet, 371 (9616), 932-944. doi: http://doi.org/10.1016/ s0140-6736(08)60419-5 
31. Vincent, A., Irani, S. R., Lang, B. (2011). Potentially pathogenic autoantibodies associated with epilepsy and encephalitis in children and adults. Epilepsia, 52, 8-11. doi: http://doi.org/10.1111/j.1528-1167.2011.03224.x

32. Granerod, J., Cunningham, R., Zuckerman, M., Mutton, K., Davies, N. W. S., Walsh, A. L. et. al. (2010). Causality in acute encephalitis: defining aetiologies. Epidemiology and Infection, 138 (6), 783-800. doi: http://doi.org/10.1017/ s0950268810000725

33. Pillai, S. C., Mohammad, S. S., Hacohen, Y., Tantsis, E., Prelog, K., Barnes, E. H. et. al. (2015). Postencephalitic epilepsy and drug-resistant epilepsy after infectious and antibody-associated encephalitis in childhood: Clinical and etiologic risk factors. Epilepsia, 57 (1), e7-e11. doi: http://doi.org/10.1111/epi.13253

34. Armangue, T., Moris, G., Cantarín-Extremera, V., Conde, C. E., Rostasy, K., Erro, M. E. et. al. (2015). Autoimmune post-herpes simplex encephalitis of adults and teenagers. Neurology, 85 (20), 1736-1743. doi: http://doi.org/10.1212/ wnl.0000000000002125

35. Bradshaw, M. J., Pawate, S., Lennon, V. A., Bloch, K. C., Brown, K. M. (2015). Herpes simplex virus 1 encephalitis associated with voltage-gated calcium channel autoimmunity. Neurology, 85 (24), 2176-2177. doi: http://doi.org/10.1212 /wnl.0000000000002218

36. Titulaer, M. J., McCracken, L., Gabilondo, I., Armangué, T., Glaser, C., Iizuka, T. et. al. (2013). Treatment and prognostic factors for long-term outcome in patients with anti-NMDA receptor encephalitis: an observational cohort study. The Lancet Neurology, 12 (2), 157-165. doi: http://doi.org/10.1016/s1474-4422(12)70310-1

37. Spatola, M., Dalmau, J. (2017). Seizures and risk of epilepsy in autoimmune and other inflammatory encephalitis. Current Opinion in Neurology, 30 (3), 345-353. doi: http://doi.org/10.1097/wco.0000000000000449

38. Murthy, J. M. K., Prabhakar, S. (2008). Bacterial meningitis and epilepsy. Epilepsia, 49, 8-12. doi: http://doi.org/ $10.1111 /$ j.1528-1167.2008.01750.x

39. Gaspard, N., Foreman, B. P., Alvarez, V., Cabrera Kang, C., Probasco, J. C. et. al. (2015). New-onset refractory status epilepticus: etiology, clinical features, and outcome. Neurology, 85, 1604-1613. doi: http://doi.org/10.1212/wnl.0000000000001940

Received date 14.08.2020

Accepted date 16.09.2020

Published date 30.11.2020

Tetyana Litovchenko, MD, Professor, Head of Department, Department of Neurology and Child Neurology, Kharkiv Medical Academy of Postgraduate Education, Amosova str., 58, Kharkiv, Ukraine, 61176 E-mail: t.litovchenko@yahoo.com

Oleksandr Bilchenko, MD, Professor, Head of Department, Department of Therapy, Nephrology and General Practice-Family Medicine, Kharkiv Medical Academy of Postgraduate Education, Amosova str., 58, Kharkiv, Ukraine, 61176

E-mail: bilchenko.post@gmail.com

Vladimir Malakhov, MD, Professor, Head of Department, Department of Medical Rehabilitation, Sports Medicine and Physical Therapy, Kharkiv Medical Academy of Postgraduate Education, Amosova str., 58, Kharkiv, Ukraine, 61176

E-mail: malakhov.reab@gmail.com

Olga Dubenko, MD, Professor, Department of Neuropathology and Neurosurgery, Kharkiv Medical Academy of Postgraduate Education, Amosova str., 58, Kharkiv, Ukraine, 61176

E-mail: Olgadubenko05@gmail.com

Olga Sukhonosova, MD, Professor, Department of Neurology and Pediatric Neurology, Kharkiv Medical Academy of Postgraduate Education, Amosova str., 58, Kharkiv, Ukraine, 61176

E-mail: vladol2017a@gmail.com

Viktoriia Bokatuieva, Researcher, Department of Vascular Pathology of the Brain and Rehabilitation, Institute of Neurology, Psychiatry and Narcology of National Academy of Medical Sciences of Ukraine, Akademika Pavlova str., 46, Kharkiv, Ukraine, 61068

E-mail: bokatueva@gmail.com

Marianna Markova, MD, Professor, Department of Sexology, Medical Psychology, Medical and Psychological Rehabilitation, Kharkiv Medical Academy of Postgraduate Education, Amosova str., 58, Kharkiv, Ukraine, 61176

E-mail: mariannochka1807@gmail.com

Vadym Nikonov, Doctor of medical sciences, professor, Head of the Department, Department of Emergency Care and Disaster Medicine, Kharkiv Medical Academy of Postgraduate Education, Amosova str., 58, Kharkiv, Ukraine, 61176

E-mail: nikonov.vad@gmail.com 